\title{
HALF-REEB COMPONENTS, PALAIS-SMALE CONDITION AND GLOBAL INJECTIVITY OF LOCAL DIFFEOMORPHISMS IN $\mathbb{R}^{3}$
}

\author{
Francisco Braun and Jean Venato-Santos
}

\begin{abstract}
Let $F=\left(F_{1}, F_{2}, F_{3}\right): \mathbb{R}^{3} \rightarrow \mathbb{R}^{3}$ be a $C^{\infty}$ local diffeomorphism. We prove that each of the following conditions are sufficient to the global injectivity of $F$ :

A) The foliations $\mathcal{F}_{F_{i}}$ made up by the connected components of the level surfaces $F_{i}=$ constant, consist of leaves without half-Reeb components induced by $F_{j}$, $j \in\{1,2,3\} \backslash\{i\}$, for $i \in\{1,2,3\}$.

B) For each $i \neq j \in\{1,2,3\},\left.F_{i}\right|_{L}: L \rightarrow \mathbb{R}$ satisfy the Palais-Smale condition, for all $L \in \mathcal{F}_{F_{j}}$.

We also prove that B) implies A) and give examples to show that the converse is not true. Further, we give examples showing that none of these conditions is necessary to the global injectivity of $F$.
\end{abstract}

2010 Mathematics Subject Classification: 37C05, 37C10, 57R30.

Key words: Half-Reeb components, foliations, Palais-Smale conditions, global injectivity.

\section{Introduction}

The problem of establishing conditions to ensure that a local diffeomorphism $F: \mathbb{R}^{n} \rightarrow \mathbb{R}^{n}$ is a diffeomorphism remounts to the beginning of the twentieth century with the work of Hadamard ([13], see also [21]) and its classical hypothesis $\int_{0}^{\infty} \inf |x| \leq s\left\|D F(x)^{-1}\right\|^{-1} d s=\infty$, or even the result of Banach and Mazur saying that $F$ is a diffeomorphism if and only if $F$ is proper (a good reference is again $[\mathbf{2 1}]$ ). Since then, many areas of Mathematics have been asking for different conditions (maybe more simple). In 1939, for example, Keller conjectured that if $F$ is polynomial, then no additional hypothesis needed to be made. This problem is known as Real Jacobian Conjecture and was proved false in 1994 by Pinchuk (see $[\mathbf{2 0}]$ ). In $\mathbb{R}^{2}$, if the degree of $F$ is low, then $F$ is a diffeomorphism (see [3]). This polynomial case of the global invertibility problem is closely related to the famous Jacobian Conjecture: very polynomial local diffeomorphism $F: \mathbb{C}^{n} \rightarrow \mathbb{C}^{n}$ is an automorphism (invertible with 
polynomial inverse). In this case, it is enough to prove the injectivity of $F$ to establish the conjecture (see $[\mathbf{2}]$ and [5]). Anyway Jacobian Conjecture remains open up to now. References can be found in [1] and in $[7]$.

Now returning to the general case ( $F$ does not to be polynomial), we can ask just for the injectivity of $F$. In $\mathbb{R}^{2}$, an interesting result is the following, due to Fernandes, Gutierrez, and Rabanal [8]:

Theorem 1.1. Let $F: \mathbb{R}^{2} \rightarrow \mathbb{R}^{2}$ be a differentiable map. If there exists $\varepsilon>0$ such that

$$
\operatorname{Spec}(F) \cap[0, \varepsilon)=\varnothing,
$$

then $F$ is globally injective.

Here $\operatorname{Spec}(F)$ stands for the set of all the (complex) eigenvalues of $D F(x)$, when $x$ varies in $\mathbb{R}^{2}$. A first $\left(C^{1}\right)$ version of this theorem appeared in $[\mathbf{9}]$, where Gutierrez solved the bi-dimensional case of the Markus-Yamabe Problem (see [16]). Indeed, in [18], Olech had already proved the equivalence between Markus-Yamabe Conjecture and the injectivity of $F$ in $\mathbb{R}^{2}$. Recently, in [12] and [22], analogous results were obtained when the equilibrium point 0 is not an attractor, but a hyperbolic saddle and a center, respectively.

The essential tool to prove Theorem 1.1 is the concept of half-Reeb component (hRc for short) of planar foliations that we recall in Definition 3.1. The connection between $\mathrm{hRc}$ and injectivity is given by Proposition 1.2 right below. But before seeing this, let us introduce a notation: given $f: M \rightarrow \mathbb{R}$ a $C^{k}$-submersion, $k \geq 1$, where $M$ is a differentiable manifold, then the connected components of the level sets of $f$ give rise to a $C^{k}$-foliation of codimension 1 of $M$. We will denote this foliation by $\mathcal{F}_{f}$.

Proposition 1.2. Let $F=\left(F_{1}, F_{2}\right): \mathbb{R}^{2} \rightarrow \mathbb{R}^{2}$ be a differentiable map such that $D F(x)$ is non singular for all $x \in \mathbb{R}^{2}$. If $F$ is not injective, then $\mathcal{F}_{F_{i}}$ has a hRc, for both $i=1,2$.

The proof of this proposition can be found in [8]. With this tool, the proof of Theorem 1.1 depicted in [8] uses Proposition 1.2 after filling in the following two items:

(i) It is shown that it is enough to prove the result under the stronger condition $\operatorname{Spec}(F) \cap(-\varepsilon, \varepsilon)=\varnothing$.

(ii) Under the spectral condition of (i), it is shown the non-existence of hRc for $\mathcal{F}_{F_{i}}, i=1,2$. 
Gutierrez and Maquera introduced in [11] the concept of half-Reeb component for a foliation $\mathcal{F}_{f}$ when $f: \mathbb{R}^{3} \rightarrow \mathbb{R}$ is a differentiable submersion. We recall this in Definition 3.4 and call it half-Reeb component of type 2 ( $\mathrm{hRc}_{2}$ for short). With this concept, they proved

Theorem 1.3. Let $F=\left(F_{1}, F_{2}, F_{3}\right): \mathbb{R}^{3} \rightarrow \mathbb{R}^{3}$ be a $C^{2}$ local diffeomorphism. If $\operatorname{Spec}(F) \cap(-\varepsilon, \varepsilon)=\varnothing$, for some $\varepsilon>0$, then $\mathcal{F}_{i}$ does not have any $\mathrm{hRc}_{2}$, for $i=1,2,3$. In particular, all the leaves of $\mathcal{F}_{F_{i}}$ are diffeomorphic to $\mathbb{R}^{2}$.

As we said above this is one of the steps to obtain the global injectivity of Theorem 1.1 in the bidimensional case (by Proposition 1.2). However, as Examples 3.5 and 3.8 below show, Proposition 1.2 is not true to maps $F: \mathbb{R}^{3} \rightarrow \mathbb{R}^{3}$, when we change hRc by $\mathrm{hRc}_{2}$. So the injectivity of $F$ can not be obtained exactly as in Theorem 1.1.

Despite this obstruction, Gutierrez and Maquera [11] used the nonexistence of $\mathrm{hRc}_{2}$ in $\mathcal{F}_{F_{i}}$, for $i=1,2,3$, to obtain a global injectivity result when $F$ is polynomial and has the set of not proper points with codimension greater than or equal to 2 . This is a weak version of the Real Jacobian Conjecture of Jelonek (see [14]) in dimension 3 and was recently generalized by Maquera and Venato-Santos in $[\mathbf{1 5}]$ to the $n$-dimensional case.

These facts motivated us to study the consequences of non-injectivity of $F=\left(F_{1}, F_{2}, F_{3}\right): \mathbb{R}^{3} \rightarrow \mathbb{R}^{3}$ on the associated foliations $\mathcal{F}_{F_{i}}$ of $\mathbb{R}^{3}$, for $i=1,2,3$. Our investigation lead us to the following: if $F$ is a not injective $C^{\infty}$ local diffeomorphism then there exist $i \neq j \in\{1,2,3\}$ and a leaf $L \in \mathcal{F}_{F_{i}}$ such that $\mathcal{F}_{\left.F_{j}\right|_{L}}$ has a hRc (observe $\mathcal{F}_{\left.F_{j}\right|_{L}}$ is a foliation of dimension 1 of $L$, since $\left.F_{j}\right|_{L}: L \rightarrow \mathbb{R}$ is a submersion). Putting this consequence more precise, let us define that $\mathcal{F}_{F_{j}}$ has a half-Reeb component of type 0 ( $\mathrm{hRc}_{0}$ for short) if there exists a leaf $L$ of one of the foliations $\mathcal{F}_{F_{i}}, i \in\{1,2,3\} \backslash\{j\}$ such that $\mathcal{F}_{\left.F_{j}\right|_{L}}$ has a hRc. With this definition, our conclusion is the following:

Theorem A. Let $F=\left(F_{1}, F_{2}, F_{3}\right): \mathbb{R}^{3} \rightarrow \mathbb{R}^{3}$ be a $C^{\infty}$ local diffeomorphism. If there is no $\mathrm{hRc}_{0}$ in the foliations $\mathcal{F}_{F_{j}}$, for all $j \in\{1,2,3\}$, then $F$ is globally injective.

Now as a consequence of Theorem A, we obtain a sufficient condition for global injectivity based on the concept of Palais-Smale (PS) condition: let $f: M \rightarrow \mathbb{R}$ be a $C^{1}$ map, where $M$ is a $C^{1}$ manifold. We say that $f$ satisfies the Palais-Smale condition (or simply PS condition) if any sequence $\left\{x_{n}\right\}$ in $M$, such that $\left\{f\left(x_{n}\right)\right\}$ is bounded and $D f\left(x_{n}\right) \rightarrow 0$, has a convergent subsequence. In the special case $D f(x) \neq 0$, for all $x \in M$, 
we can state $f$ satisfies PS condition when for any sequence $\left\{x_{n}\right\}$, such that $x_{n} \rightarrow \infty$ and $f\left(x_{n}\right) \rightarrow c,{ }^{1}$ there exists $\varepsilon>0$ with $\left\|D f\left(x_{n}\right)\right\| \geq \varepsilon$, for all $n \in \mathbb{N}$, where $\|\cdot\|$ is a Riemannian Metric of $M$. The use of PS type condition as a mechanism to globalize the injectivity of local diffeomorphisms has been exploited in many recent works, see for instance $[\mathbf{4}, \mathbf{1 0}, \mathbf{1 7}, \mathbf{2 3}, \mathbf{2 4}, \mathbf{2 7}]$ and the references therein. Theorem $\mathrm{B}$ bellow generalizes to $\mathbb{R}^{3}$ the bidimensional result of $[\mathbf{1 0}]$ : "Given $F: \mathbb{R}^{2} \rightarrow \mathbb{R}^{2}$ a $C^{\infty}$ local diffeomorphism, if $F_{i}: \mathbb{R}^{2} \rightarrow \mathbb{R}$ satisfies the PS condition, then $\mathcal{F}_{F_{i}}$ has no hRc". Then Proposition 1.2 gives the global injectivity of $F$.

Theorem B. Let $F=\left(F_{1}, F_{2}, F_{3}\right): \mathbb{R}^{3} \rightarrow \mathbb{R}^{3}$ be a $C^{\infty}$ local diffeomorphism. If for each $i \neq j \in\{1,2,3\},\left.F_{i}\right|_{L}: L \rightarrow \mathbb{R}$ satisfy PS condition, for all $L \in \mathcal{F}_{F_{j}}$, then $F$ is globally injective.

The paper is organized as follows. In Section 2, we recall the known concept of global solvability of vector fields and see a known result of global injectivity using this concept, which is a different generalization of Proposition 1.2 in all dimensions.

In the third section, we recall the definitions of hRc in a 2 manifold and $h R c_{2}$ in $\mathbb{R}^{3}$. We also give examples that motivate the definitions of $h \mathrm{hc}_{1}$ and $h \mathrm{hc}_{0}$, and Theorem A.

Finally, Section 4 is devoted to prove Theorem A after proving the more general Propositions 4.1 and 4.2. In fact the last one guarantees that $\mathrm{hRc}_{2}$ implies the existence of $\mathrm{hRc}_{0}$. We then finish with the proof of Theorem B, which is a direct consequence of Theorem A and Proposition 4.4, where we prove (adapting ideas of [10]) that the existence of hRc in $\mathcal{F}_{\left.F_{i}\right|_{L}}, L \in \mathcal{F}_{F_{j}}$, guarantees that $\left.F_{i}\right|_{L}: L \rightarrow \mathbb{R}$ does not satisfy PS condition.

The very simple Example 3.11 shows that the reciprocal of Proposition 4.4 is not true and that the condition in Theorem B is not necessary for injectivity.

\section{Preliminaries}

We now recall the definition of global solvability of vector fields.

Definition 2.1. Let $M$ be a $C^{\infty}$ manifold and $X: C^{\infty}(M) \rightarrow C^{\infty}(M)$ be a vector field. We say that $X$ is globally solvable when $X$ is surjective, i.e. given $f: M \rightarrow \mathbb{R}$, there is $u: M \rightarrow \mathbb{R}$ such that $X u=f$ ( $f$ and $u$ are $\left.C^{\infty}\right)$.

${ }^{1} x_{n} \rightarrow \infty$ means that $\left\{x_{n}\right\}$ does not have any convergent subsequence. 
The next result gives a geometric characterization of global solvability and is part of Theorem 6.4.2 of [6], due to Duistermaat and Hörmander.

Lemma 2.2. Let $M$ be a $C^{\infty}$ manifold and $X: C^{\infty}(M) \rightarrow C^{\infty}(M)$ be a vector field. The items bellow are equivalent:

(1) $X$ is globally solvable.

(2) (a) No integral curve of $X$ is contained in a compact subset of $M$.

(b) For all compact $K \subset M$, there exists a compact $K^{\prime} \subset M$ such that every compact interval on an integral curve of $X$ with end points in $K$ is contained in $K^{\prime}$.

Now given a $C^{\infty}$ map $F=\left(F_{1}, \ldots, F_{n}\right): \mathbb{R}^{n} \rightarrow \mathbb{R}^{n}$, we define $n$ vector fields $\nu_{i}, i=1, \ldots, n$, as follows:

$$
\nu_{i}(\phi)=\operatorname{det} D\left(F_{1}, \ldots, F_{i-1}, \phi, F_{i+1}, \ldots, F_{n}\right),
$$

and recall the following result of $[\mathbf{2 6}]$ (see also [25]):

Theorem 2.3. Let $F: \mathbb{R}^{n} \rightarrow \mathbb{R}^{n}$ be a $C^{\infty}$ local diffeomorphism. If $\nu_{i}$ is globally solvable for $n-1$ different indices $i \in\{1, \ldots, n\}$, then $F$ is injective.

In the next result, we will see some useful properties of the vector fields $\nu_{i}$.

Lemma 2.4. Let $F: \mathbb{R}^{n} \rightarrow \mathbb{R}^{n}$ be a $C^{\infty}$ map such that $\operatorname{det} D F(x) \neq 0$, $\forall x \in \mathbb{R}^{n}$. For each $i \in\{1, \ldots, n\}$, the following holds:

(1) The integral curves of $\nu_{i}$ are the non-empty connected components of the intersections

$$
\cap_{\substack{j=1 \\ j \neq i}}^{n} F_{j}^{-1}\left(c_{j}\right), \quad c_{j} \in \mathbb{R} .
$$

(2) $F_{i}$ is strictly monotone along the integral curves of $\nu_{i}$.

(3) The alpha and omega limit sets of each integral curve of $\nu_{i}$ are empty.

Proof: Given $\gamma(t)$ an integral curve of $\nu_{i}$, we have that for each $j \in$ $\{1, \ldots, n\},\left(F_{j}(\gamma)\right)^{\prime}(t)=\delta_{i j} \operatorname{det} D F(\gamma(t))$, where $\delta_{i j}$ is the Kronecker delta. This shows that $F_{i}$ is strictly monotone along $\gamma$ (proving item (2)) and that $\gamma$ is contained in a connected component of one of the intersections of item (1). Since these connected components are $C^{\infty}$ curves (by the Implicit Function Theorem), we have by maximality of $\gamma(t)$ that it must coincide with this curve, proving item (1). To prove item (3), observe that item (1) guarantees that each integral curve of $\nu_{i}$ is a closed set, so it contains its alpha and omega limit sets. If the alpha (or the omega) limit set of one integral curve of $\nu_{i}$ is non-empty, we will have a periodic integral curve, what is impossible by item (2). 


\section{Examples and half-Reeb components of types 0,1 and 2}

We start this section recalling the concepts of $h R c$ in foliations of two-dimensional manifolds and $\mathrm{hRc}_{2}$ in foliations of $\mathbb{R}^{3}$.

Definition 3.1. Let $M$ be a $C^{\infty}$ manifold of dimension 2 and $f: M \rightarrow \mathbb{R}$ be a $C^{\infty}$ submersion. We say that $\mathcal{A} \subset M$ is a half-Reeb component (or simply a hRc) of $\mathcal{F}_{f}$ if there is a homeomorphism $G: B \rightarrow \mathcal{A}$ which is a topological equivalence between $\left.\mathcal{F}_{f}\right|_{\mathcal{A}}$ and $\left.\mathcal{F}_{f_{0}}\right|_{B}$, where $B=\{(x, y) \in$ $[0,2] \times[0,2] ; 0<x+y \leq 2\}$ and $f_{0}: \mathbb{R}^{2} \rightarrow \mathbb{R}$ is defined by $f_{0}(x, y)=x y$. Moreover, $G$ satisfies

(1) The segment $\{(x, y) \in B \mid x+y=2\}$ is sent by $G$ onto a curve which gives two transversal sections for the foliation $\mathcal{F}_{f}$ in the complement of $G(1,1)$; this curve is called the compact edge of $\mathcal{A}$.

(2) Both segments $\{(x, y) \in B \mid x=0\}$ and $\{(x, y) \in B \mid y=0\}$ are sent by $G$ onto full half-trajectories of $\mathcal{F}_{f}$. These two half-trajectories of $\mathcal{F}_{f}$ are called the non-compact edges of $\mathcal{A}$.

Remark 3.2. Note that, given a $C^{\infty}$ map $F=\left(F_{1}, F_{2}\right): \mathbb{R}^{2} \rightarrow \mathbb{R}^{2}$, such that $\operatorname{det} D F(x) \neq 0, \forall x \in \mathbb{R}^{2}$, each connected component of the intersections given by Lemma 2.4, for $i=1$ or 2, are exactly the leaves of the foliations $\mathcal{F}_{F_{2}}$ or $\mathcal{F}_{F_{1}}$, respectively. Then Lemma 2.2 shows that $\mathcal{F}_{F_{i}}$ does not have any hRc if and only if $\nu_{j}$ is globally solvable, for $i \neq j \in\{1,2\}$. So in $\mathbb{R}^{2}$, Theorem 2.3 and Proposition 1.2 are equivalent. ${ }^{2}$

Now the $\mathrm{hRc}_{2}$ concept (according to $[\mathbf{1 1}]$ ):

Definition 3.3. Let $f: \mathbb{R}^{3} \rightarrow \mathbb{R}$ be a $C^{2}$ submersion. We say that a $C^{2}$-embedding $H_{0}: S^{1} \rightarrow \mathbb{R}^{3}$ is a vanishing cycle for $\mathcal{F}_{f}$ if it satisfies:

(1) $H_{0}\left(S^{1}\right)$ is contained in a leaf $L_{0}$ but is not homotopic to a point in $L_{0}$.

(2) $H_{0}$ can be extended to a $C^{2}$-embedding $H:[-1,2] \times S^{1} \rightarrow \mathbb{R}^{3}$ such that for all $t \in(0,1]$, there is a 2-disc $D_{t}$ contained in a leaf $L_{t} \in \mathcal{F}_{f}$ with $\partial D_{t}=H\left(\{t\} \times S^{1}\right)$.

(3) For all $x \in S^{1}$, the curve $t \in[-1,2] \mapsto H(t, x)$ is transverse to the foliation $\mathcal{F}_{f}$ and, for all $t \in(0,1]$, the disc $D_{t}$ depends continuously on $t$.

We say that the leaf $L_{0}$ supports the vanishing cycle $H_{0}$ and that the map $H$ is associated to $H_{0}$.

\footnotetext{
${ }^{2}$ Observe that when $n=2, \nu_{i}=(-1)^{i} H_{F_{j}}, i \neq j \in\{1,2\}$, where $H_{F_{j}}$ stands for the Hamiltonian vector field associated to $F_{j}, H_{F_{j}}=-\partial_{2} F_{j} \partial_{1}+\partial_{1} F_{j} \partial_{2}$.
} 
Definition 3.4. A half-Reeb component of type 2 (that we denote by $\mathrm{hRc}_{2}$ ) of $\mathcal{F}_{f}$ associated to the vanishing cycle $H_{0}$ is the region

$$
\mathcal{A}=\left(\bigcup_{t \in(0,1]} D_{t}\right) \cup L \cup H_{0}\left(S^{1}\right),
$$

where $L$ is the connected component of $L_{0} \backslash H_{0}\left(S^{1}\right)$ contained in the closure of $\cup_{t \in(0,1]} D_{t}$. We say that the transversal $H\left([0,1] \times S^{1}\right)$ (to the foliation $\mathcal{F}_{f}$ ) is the compact face of $\mathcal{A}$ and the half-leaf $L \cup H_{0}\left(S^{1}\right)$ is the non-compact face of $\mathcal{A}$.

As we said in the introduction, just the non-existence of $\mathrm{hRc}_{2}$ in the foliations $\mathcal{F}_{F_{i}}, i=1,2,3$ does not imply the injectivity of $F$ in dimension 3. Here we give some examples:

Example 3.5. Consider the map $F: \mathbb{R}^{3} \rightarrow \mathbb{R}^{3}$ defined by

$$
F\left(x_{1}, x_{2}, x_{3}\right)=\left(F_{1}\left(x_{1}, x_{2}\right), F_{2}\left(x_{1}, x_{2}\right), x_{3}\right),
$$

where $\left(F_{1}, F_{2}\right): \mathbb{R}^{2} \rightarrow \mathbb{R}^{2}$ is a differentiable non-injective local diffeomorphism (for example, take $F_{1}=e^{x_{1}} \cos \left(x_{2}\right)$ and $F_{2}=e^{x_{1}} \sin \left(x_{2}\right)$ ). The leaves of the foliations $\mathcal{F}_{F_{i}}$, for $i=1,2,3$, are all diffeomorphic to the plane, so it is clear that each of these foliations has a $\mathrm{hRc}_{2}$.

More precisely, the leaves of the foliation $\mathcal{F}_{F_{3}}$ are the planes $x_{3}=c$, $c \in \mathbb{R}$. Now the leaves of $\mathcal{F}_{F_{i}}$, for $i=1$ or 2 are of the form $l \times \mathbb{R}$, where $l$ are the leaves of the foliations of $\mathbb{R}^{2}, \mathcal{F}_{F_{1}}$ and $\mathcal{F}_{F_{2}}$, respectively.

It is simple to modify the map of the example above in a way that one of the foliations $\mathcal{F}_{F_{i}}$ presents $\mathrm{hRc}_{2}$ :

Example 3.6. Consider the map $F: \mathbb{R}^{3} \rightarrow \mathbb{R}^{3}$ defined by

$$
F\left(x_{1}, x_{2}, x_{3}\right)=\left(F_{1}\left(x_{1}, x_{2}\right), F_{2}\left(x_{1}, x_{2}\right), x_{1}^{2}+x_{2}^{2}-e^{x_{3}}\right),
$$

where $\left(F_{1}, F_{2}\right): \mathbb{R}^{2} \rightarrow \mathbb{R}^{2}$ is a differentiable non-injective local diffeomorphism. The foliations $\mathcal{F}_{F_{i}}$, for $i=1$ and 2 have the same property than the ones of Example 3.5. But the foliation $\mathcal{F}_{F_{3}}$ has a $\mathrm{hRc}_{2}$.

In these two examples, as we said, the foliations $\mathcal{F}_{F_{i}}$, for $i=1,2$, have its leaves of the form $l \times \mathbb{R}$, where $l$ are the leaves of the foliation $\mathcal{F}_{F_{i}}$, respectively. Since the map $\left(x_{1}, x_{2}\right) \mapsto\left(F_{1}\left(x_{1}, x_{2}\right), F_{2}\left(x_{1}, x_{2}\right)\right)$ is not injective, we get by Proposition 1.2 that $\mathcal{F}_{F_{i}}$, for $i=1$ and 2 , exhibit hRc.

Let $f: \mathbb{R}^{3} \rightarrow \mathbb{R}$ be a $C^{\infty}$ submersion such that all the leaves of $\mathcal{F}_{f}$ are diffeomorphic to $\mathbb{R}^{2}$ (so $\mathcal{F}_{f}$ does not have $\mathrm{hRc}_{2}$ ). By Corollary 1 of $[\mathbf{1 9}]$, there is a diffeomorphism $H: \mathbb{R}^{3} \rightarrow \mathbb{R}^{3}$ that take leaves of $\mathcal{F}_{f}$ 
onto leaves of a foliation of type $\mathcal{F}_{0} \times \mathbb{R}$, where $\mathcal{F}_{0}$ is a foliation of $\mathbb{R}^{2}$. We say that $\mathcal{F}_{f}$ has a half-Reeb component of type 1 , (or simply $\mathrm{hRc}_{1}$ ) if $\mathcal{F}_{0}$ has a hRc (it is possible to prove that $\mathcal{F}_{0}$ is defined by the connected components of a submersion - see the second part of Remark 3.7). See Figure 1(b).

Remark 3.7. Let $f: \mathbb{R}^{3} \rightarrow \mathbb{R}$ be a $C^{\infty}$ submersion such that all the leaves of $\mathcal{F}_{f}$ are diffeomorphic to $\mathbb{R}^{2}$. Then $\mathcal{F}_{f}$ has a $h R c_{1}$ if, and only if, $f$ has a disconnected level set. Indeed, suppose first that $\mathcal{F}_{f}$ has a $\mathrm{hRc}_{1}$ and consider $H: \mathbb{R}^{3} \rightarrow \mathbb{R}^{3}$ and $\mathcal{F}_{0}$ as in the definition above. Since $\mathcal{F}_{0}$ has a hRc, there are points $a, b \in \mathbb{R}^{2}$ in different leaves of $\mathcal{F}_{0}$ and a sequence $l_{n}$ of leaves of $\mathcal{F}_{0}$ each of them containing $a_{n}, b_{n}$ points of $\mathbb{R}^{2}$ such that $a_{n} \rightarrow a$ and $b_{n} \rightarrow b$. Since $f\left(H^{-1}\left(a_{n}, 0\right)\right)=f\left(H^{-1}\left(b_{n}, 0\right)\right)$, we get by continuity that $H^{-1}(a, 0)$ and $H^{-1}(b, 0)$ are in the same level set of $f$. This level set must be disconnected (since its image by $H$ is disconnected).

On the other hand, since the leaves of $\mathcal{F}_{f}$ are all diffeomorphic to $\mathbb{R}^{2}$, we have by Corollary 1 of $[\mathbf{1 9}]$ that there is $H: \mathbb{R}^{3} \rightarrow \mathbb{R}^{2} \times \mathbb{R}$ a diffeomorphism such that $\mathcal{F}_{f}$ is taken by $H$ in a foliation of type $\mathcal{F}_{0} \times \mathbb{R}$, where $\mathcal{F}_{0}$ is a foliation of $\mathbb{R}^{2}$. Then it is not difficult to see that $\mathcal{F}_{0}$ is the foliation $\mathcal{F}_{g}$ where $g: \mathbb{R}^{2} \rightarrow \mathbb{R}$ is the submersion defined by $g(x)=f\left(H^{-1}(x, 0)\right)$. If $f$ has a disconnected level set then so does $g$. Then (by Theorem 2.4 of [3], for example, or, for a different argument, see the proof of Proposition 1.4 of $[8]), \mathcal{F}_{0}=\mathcal{F}_{g}$ has a hRc and so $\mathcal{F}_{f}$ has a hRc $\mathrm{h}_{1}$ by definition.

With this concept, we ask if the non injectivity of $F: \mathbb{R}^{3} \rightarrow \mathbb{R}^{3}$ implies that one of the foliations $\mathcal{F}_{F_{i}}$ has $\mathrm{hRc}_{1}$ or $\mathrm{hRc}_{2}$. Next example shows it is not the case:

Example 3.8. Let $F: \mathbb{R}^{3} \rightarrow \mathbb{R}^{3}$ be defined by

$$
F\left(x_{1}, x_{2}, x_{3}\right)=\left(x_{3}-e^{x_{1}} \cos x_{2}, x_{3}-e^{x_{1}} \sin x_{2}, x_{3}\right) .
$$

$F$ is clearly not injective and $\operatorname{det} D F(x)=e^{2 x_{1}}$.

Moreover, all the level sets of $F_{i}$ are clearly connected and diffeomorphic to $\mathbb{R}^{2}$, for $i=1,2,3$ (all of them are graphics of $C^{\infty}$ maps carrying $\mathbb{R}^{2}$ to $\mathbb{R}$ ). Hence, the foliations $\mathcal{F}_{F_{i}}, i=1,2,3$, do not have $\mathrm{hRc}_{2}$ neither $\mathrm{hRc}_{1}$ (by Remark 3.7).

Remark 3.9. Example 3.8 above also shows that $F_{i}: \mathbb{R}^{3} \rightarrow \mathbb{R}$ satisfying PS condition, for $i=1,2,3$, is not sufficient for global injectivity. Indeed, we have that $\left\|\nabla F_{i}(x)\right\| \geq 1$, for all $i=1,2,3$. 
So, what is the influence of the non-injectivity of $F$ on the foliations $\mathcal{F}_{F_{i}}$ ? Observe that in the three examples above we have the (opposite) property of our main Theorem A: in Example 3.5, any leaf $L$ of $\mathcal{F}_{F_{3}}$ satisfies that $\mathcal{F}_{\left.F_{i}\right|_{L}}$ have hRc for $i=1$,2. In Example 3.6, take the leaves $L=F_{3}^{-1}(c)$, with $c<0$, of $\mathcal{F}_{F_{3}}$. It is also clear that $\mathcal{F}_{\left.F_{i}\right|_{L}}$ have hRc for $i=1,2$. Now in Example 3.8, take $L=F_{3}^{-1}(0)$ the leaf of $\mathcal{F}_{F_{3}}$ to be considered. It is also simple to see that $\mathcal{F}_{\left.F_{i}\right|_{L}}$ have $\mathrm{hRc}$, for $i=1,2$. So the common property is that at least one of the foliations $\mathcal{F}_{F_{i}}$ has a $\mathrm{hRc}_{0}$ as defined in the introduction.

See Figure 1 for the $h R c_{0}, h R c_{1}$ and $h R c_{2}$ in $\mathbb{R}^{3}$.

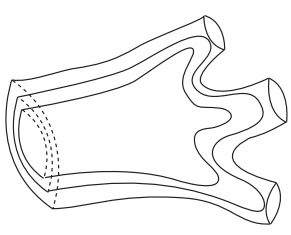

(a) $\mathrm{hRc}_{2}$

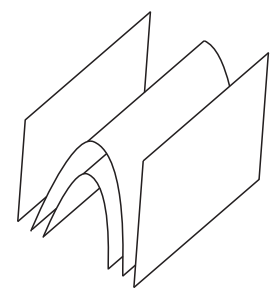

(b) $\mathrm{hRc}_{1}$

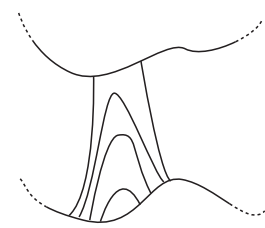

(c) $\mathrm{hRc}_{0}$

\section{Figure 1.}

Remark 3.10. In the next section we will prove, in Proposition 4.2, that the existence of $\mathrm{hRc}_{2}$ implies the occurrence of $\mathrm{hRc}_{0}$. Hence, modifying Example 3.6 such that $F_{1}\left(x_{1}, x_{2}\right)=x_{1}$ and $F_{2}\left(x_{1}, x_{2}\right)=x_{2}$, for example, we have that $\mathcal{F}_{F_{3}}$ has a $\mathrm{hRc} \mathrm{c}_{0}$ despite of the injectivity of $F$. Proving that the non existence of $\mathrm{hRc}_{0}$ is not necessary to the injectivity in $n=3$.

Example 3.11. Let $F: \mathbb{R}^{3} \rightarrow \mathbb{R}^{3}$ be defined by

$$
F\left(x_{1}, x_{2}, x_{3}\right)=\left(\arctan x_{1}, x_{2}, x_{3}\right),
$$

where we choose the values of $\arctan x_{1}$ in the interval $(-\pi / 2, \pi / 2)$. It is clear that $\operatorname{det} D F(x)=\frac{1}{1+x_{1}^{2}}>0$ and $F$ is globally injective. Moreover, there is no $\mathrm{hRc}_{0}$ in the foliations $\mathcal{F}_{F_{j}}$, for all $j \in\{1,2,3\}$.

Now, consider the leaf $L=\mathbb{R} \times\{0\} \times \mathbb{R}$ of $\mathcal{F}_{F_{2}}$ and the sequence $p_{n}=$ $(n, 0,0) \rightarrow \infty$ in $L$. We have that $F_{1}\left(p_{n}\right) \rightarrow \pi / 2$ and $D\left(\left.F_{1}\right|_{L}\right)\left(p_{n}\right)=$ $\left(\frac{1}{1+n^{2}}, 0,0\right) \rightarrow 0$, when $n \rightarrow \infty$. That is, $\left.F_{1}\right|_{L}$ does not satisfies PS condition. Indeed, it is simple to observe that $\left.F_{1}\right|_{M}$ does not satisfy PS condition for any $M \in \mathcal{F}_{F_{j}}, j=2$ and 3 . This shows that condition A) is weaker than B). 


\section{Proof of main results}

We will prove the following stronger proposition, which by Theorem 2.3 and Proposition 4.2 will result in Theorem A.

Proposition 4.1. Let $F: \mathbb{R}^{3} \rightarrow \mathbb{R}^{3}$ be a $C^{\infty}$ local diffeomorphism. If there is $i \in\{1,2,3\}$ such that $\nu_{i}$ is not globally solvable, then there is $j \in\{1,2,3\} \backslash\{i\}$ such that $\mathcal{F}_{F_{j}}$ has a $\mathrm{hRc}_{0}$. More precisely, there is $L \in \mathcal{F}_{F_{k}}$, with $k \in\{1,2,3\} \backslash\{i, j\}$, such that $\mathcal{F}_{F_{j} \mid L}$ has a hRc.

Proof: Let us suppose without loss of generality that $\nu_{3}$ is not globally solvable.

We will first make the proof supposing that $\mathcal{F}_{F_{1}}$ and $\mathcal{F}_{F_{2}}$ are foliations whose leaves are diffeomorphic to $\mathbb{R}^{2}$.

By Lemma 2.2 there exists a compact $K \subset \mathbb{R}^{3}$ such that for all $n \in \mathbb{N}$, there exist $\gamma_{n}$, an integral curve of $\nu_{3}$, and $0<s_{n}<t_{n} \in \mathbb{R}$ such that $\gamma_{n}(0), \gamma_{n}\left(t_{n}\right) \in K$ but $\left|\gamma_{n}\left(s_{n}\right)\right|>n$. Since $K$ is compact, passing to subsequences, if necessary, we may assume that $\gamma_{n}(0) \rightarrow a \in K$ and $\gamma_{n}\left(t_{n}\right) \rightarrow b \in K$. Furthermore, since $\gamma_{n}(0), \gamma_{n}\left(t_{n}\right) \in F_{1}^{-1}\left\{c_{1 n}\right\} \cap$ $F_{2}^{-1}\left\{c_{2 n}\right\}$, for some $c_{1 n}, c_{2 n} \in \mathbb{R}$ (by Lemma 2.4), we have that $a$ and $b$ are in the same level set of both $F_{1}$ and $F_{2}$. Let us denote $L_{a}^{i}$ and $L_{b}^{i}$ the leaves of $\mathcal{F}_{F_{i}}$ which contain $a$ and $b$, respectively, for $i=1,2$. We have two possibilities:

(1) $L_{a}^{1}=L_{b}^{1}$ or $L_{a}^{2}=L_{b}^{2}$;

(2) $L_{a}^{1} \neq L_{b}^{1}$ and $L_{a}^{2} \neq L_{b}^{2}$.

In case (1), suppose that $L_{a}^{2}=L_{b}^{2}$. We assert $a$ and $b$ can not be in the same integral curve of $\nu_{3}$, since if this is so, by the Flow Box Theorem, we can construct a tubular neighborhood $\Gamma$ along the arc of integral curve $\gamma$ from $a$ to $b$. Since each leaf of $\mathcal{F}_{F_{3}}$ is a local transversal section of $\nu_{3}$, this tubular neighborhood can be built such that the leaf of $\mathcal{F}_{F_{3}}$ passing through $a$ is a global transversal section of $\left.\nu_{3}\right|_{\Gamma}$. Then by item (2) of Lemma 2.4, we have that each integral curve of $\nu_{3}$ enters $\Gamma$ just once. So for $n$ big enough, the arcs of trajectories $\gamma_{n}$ from $\gamma_{n}(0)$ to $\gamma_{n}\left(t_{n}\right)$ are entirely contained in $\Gamma$. But this is a clear contradiction with the fact stated above that there exists $s_{n}$ in $\left(0, t_{n}\right)$ such that $\left|\gamma\left(s_{n}\right)\right| \rightarrow \infty$.

This assertion then issues that $\left.F_{1}\right|_{L_{a}^{2}}: L_{a}^{2} \rightarrow \mathbb{R}$ is a submersion with a disconnected level set (again by Lemma 2.4). We then take $h: L_{a}^{2} \rightarrow \mathbb{R}^{2}$ a diffeomorphism and consider the submersion $f: \mathbb{R}^{2} \rightarrow \mathbb{R}$ given by $f=F_{1} \circ h^{-1}$. It is clear $f$ has a disconnected level set and so $\mathcal{F}_{f}$ has a hRc (again by Theorem 2.4 of [3] or by the proof of Proposition 1.4 of $[8])$. So by definition, $\mathcal{F}_{\left.F_{1}\right|_{L_{a}^{2}}}$ has a hRc. Consequently, $\mathcal{F}_{F_{1}}$ has a $\mathrm{hRc}_{0}$. 
Now for case (2), call $L_{n}^{1}$ and $L_{n}^{2}$ the leaves of $\mathcal{F}_{F_{1}}$ and $\mathcal{F}_{F_{2}}$, which contains $\gamma_{n}$, respectively. We assert there is $n_{0}$ such that for all $n \geq n_{0}$, $L_{n}^{2} \cap L_{a}^{1} \neq \varnothing$ and $L_{n}^{2} \cap L_{b}^{1} \neq \varnothing$.

Before proceeding to the proof of the assertion, we observe it already implies the existence of hRc of $\mathcal{F}_{\left.F_{1}\right|_{L_{n}^{2}}}$ on each of the leaves $L_{n}^{2}$ (i.e. $\mathcal{F}_{F_{1}}$ has a $h R c_{0}$ ), for all $n \geq n_{0}$, by the same reason above, since $\left.F_{1}\right|_{L_{n}^{2}}: L_{n}^{2} \rightarrow$ $\mathbb{R}$ has a disconnected level set.

So let us prove the assertion. In a neighborhood of $a$, we can trivialize the foliation $\mathcal{F}_{F_{2}}$. So since $L_{a}^{1}$ intersects transversally $L_{a}^{2}$, this transversal intersection also occurs with leaves of $\mathcal{F}_{F_{2}}$ which contain points near enough of $a$. Since $\gamma_{n}(0) \rightarrow a$ as $n \rightarrow \infty$, it is clear $L_{n}^{2}$ will intersect transversally $L_{a}^{1}$ for $n$ big enough. The same can be done to show $L_{n}^{2} \cap$ $L_{b}^{1} \neq \emptyset$, as we wanted.

Now in the case there is a leaf of one of the foliations $\mathcal{F}_{F_{1}}$ or $\mathcal{F}_{F_{2}}$ which is not diffeomorphic to $\mathbb{R}^{2}$, Proposition 2.2 of [11] asserts the existence of a hRc $c_{2}$ of the foliation $\mathcal{F}_{F_{1}}$ or $\mathcal{F}_{F_{2}}$, respectively. The proof then follows by next result.

Proposition 4.2. Let $F: \mathbb{R}^{3} \rightarrow \mathbb{R}^{3}$ be a $C^{1}$ local diffeomorphism such that $\mathcal{F}_{F_{i}}$ has a $\mathrm{hRc}_{2}$, for some $i \in\{1,2,3\}$. Then $\mathcal{F}_{F_{i}}$ has a $\mathrm{hRc}_{0}$. More precisely, for each $k \in\{1,2,3\} \backslash\{i\}$, there exists a leaf $L \in \mathcal{F}_{F_{k}}$ such that $\mathcal{F}_{\left.F_{i}\right|_{L}}$ has a hRc.

Proof: Let us suppose without loss of generality that $i=1$ and that $k=2$. Consider so $\mathcal{A}$ a $\mathrm{hRc}_{2}$ of $\mathcal{F}_{F_{1}}$. In our arguments we will use the notations of Definitions 3.3 and 3.4.

For each $n$, choose $x_{n} \in D_{1 / n}$ in such a way that $x_{n} \rightarrow \infty$ when $n \rightarrow$ $\infty$. Consider $\gamma_{n}$ the integral curve of the vector field $\nu_{3}$ through $x_{n}$. This curve will cut the compact edge of $\mathcal{A}$ in two points $a_{n}, b_{n}$, for each $n \in \mathbb{N}$. It is clear that $a_{n} \rightarrow a$ and $b_{n} \rightarrow b$, where $a$ and $b$ are points in the evanescent cycle of $\mathcal{A}$. Take now $L_{a}^{2}$ the leaf of $\mathcal{F}_{F_{2}}$ passing trough $a$. We assert that the intersection of $L_{a}^{2}$ with $L_{0}$ will give at least two connected components, i.e. at least two distinct integral curves of $\nu_{3}$. Moreover, each of these integral curves has one end entirely contained in $L$. Indeed, let us first prove that one end of the integral curve of $\nu_{3}$ passing through $a, \gamma_{a}$, is entirely contained in $L$. This is so because if there is a compact arc of trajectory of $\gamma_{a}$ in $L$ with ends in the evanescent cycle of $\mathcal{A}$, then we use the Flow Box Theorem, as in the proof of last proposition, to construct a tubular neighborhood along this arc and get a contradiction with the fact that $x_{n} \rightarrow \infty$ (recall that the integral curves of $\nu_{3}$ are connected components of the intersections of the level sets of $F_{1}$ and $F_{2}$ by Lemma 2.4). Now to finish the proof of the assertion, observe 
that $L_{a}^{2}$ has to intersect the evanescent cycle of $\mathcal{A}$ in at least another point different of $a$. Then repeat the argument for this point.

Let us suppose, without loss of generality, that there is an $\operatorname{arc} \beta$ in the evanescent cycle connecting $a$ and $b$ such that $\beta \cap L_{a}^{2}=\{a, b\}$. So the intersection of $L_{a}^{2}$ with the compact face of $\mathcal{A}$ contains two curves $\alpha_{a}(t)$ and $\alpha_{b}(t)$ in such a way that $\alpha_{a}(0)=a$ and $\alpha_{b}(0)=b$. Moreover, $\alpha_{a}(t)$ and $\alpha_{b}(t)$ are points in $\partial D_{t}$ with the property that the connected component of the intersection of $L_{a}^{2}$ with $D_{t}$ containing the point $\alpha_{a}(t)$ is a curve with ends $\alpha_{a}(t)$ and $\alpha_{b}(t)$, for $t \in(0, c]$, for some $c \in(0,1)$. Let us call this curve $\gamma_{t}$. We will suppose, without loss of generality that $c=1 / 2$.

By construction, $\alpha_{a}$ and $\alpha_{b}$ are transversal sections of the foliation given by the connected components of $D_{t} \cap L_{a}^{2}, t \in(0,1 / 2)$.

Consider now $B=\{(x, y) \in[0,2] \times[0,2] \mid 0<x+y \leq 2\}$ and $f_{0}: B \rightarrow \mathbb{R}$ given by $f_{0}(x, y)=x y$, as in Definition 3.1. It is clear we can define a $C^{1}$ map $H: B \backslash\left\{(x, y) \mid f_{0}(x, y)=s, s \in(1 / 2,1]\right\} \rightarrow L_{a}^{2}$ satisfying the following:

(1) $H\left(f_{0}^{-1}(t(2-t))\right)=\gamma_{t}$, for each $t \in(0,1 / 2]$,

(2) $H(\{0\} \times(0,2))=\gamma_{a}$ and $H((0,2) \times\{0\})=\gamma_{b}$, where $\gamma_{a}$ and $\gamma_{b}$ are the half-trajectories of $\nu_{3}$ passing through $a$ and $b$, respectively, and contained in $L_{0}$.

Denote $\mathcal{C}=H\left(B \backslash\left\{(x, y) \mid f_{0}(x, y)=s, s \in(1 / 2,1]\right\}\right)$. Now by a composition, we clearly obtain $G: B \rightarrow \mathcal{C}$ satisfying Definition 3.1. So $\mathcal{C}$ is a hRc of $\mathcal{F}_{\left.F_{1}\right|_{L_{a}^{2}}}$, i.e. we have that $\mathcal{F}_{F_{1}}$ has a $\mathrm{hRc} \mathrm{c}_{0}$.

Before proving Theorem B, let us make some remarks.

Given a $C^{1}$ map $f: \mathbb{R}^{3} \rightarrow \mathbb{R}$, let $\nabla f: \mathbb{R}^{3} \rightarrow \mathbb{R}^{3}$ denote the usual gradient vector of $f$, that is $\nabla f=\left(\partial_{1} f, \partial_{2} f, \partial_{3} f\right)$. Let $L$ be a 2-dimensional $C^{1}$-submanifold of $\mathbb{R}^{3}$. It is clear that $D\left(\left.f\right|_{L}\right)(p)$ is the restriction of $\nabla f(p)$ to the subspace $T_{p} L$, for each $p \in L$. In other words, it is the projection of $\nabla f(p)$ at the tangent space $T_{p} L$, for each $p \in L$.

Remark 4.3. If $F: \mathbb{R}^{3} \rightarrow \mathbb{R}^{3}$ is a $C^{1}$ local diffeomorphism and $L$ is a leaf of $\mathcal{F}_{F_{j}}$, for some $j=1,2$ or 3 , then $D\left(\left.F_{i}\right|_{L}\right)(p) \neq 0$, for all $p \in L$ and $i \neq j$. In fact, since $F$ is a local diffeomorphism, $\left\{\nabla F_{i}(p) \mid i=1,2,3\right\}$ is a basis for $\mathbb{R}^{3}$, for each $p \in \mathbb{R}^{3}$. Then since $\nabla F_{j}(p)$ is normal to $T_{p} L$, we conclude that $D\left(\left.F_{i}\right|_{L}\right)(p) \neq 0$, for all $p \in L$.

It is simple to see that in Example 3.8 above we have $F_{i}$ satisfying PS condition for $i=1,2$ and 3. So this is not sufficient for global injectivity. On the other hand, the next result with Theorem A show that the new PS condition introduced above is sufficient (and so prove Theorem B): 
Proposition 4.4. Let $F=\left(F_{1}, F_{2}, F_{3}\right): \mathbb{R}^{3} \rightarrow \mathbb{R}^{3}$ be a $C^{1}$ local diffeomorphism. If $\left.F_{i}\right|_{L}: L \rightarrow \mathbb{R}$ satisfy $P S$ condition, where $L$ is a leaf of $\mathcal{F}_{F_{j}}$, for $j \neq i \in\{1,2,3\}$, then $\mathcal{F}_{F_{i}}$ has no $\mathrm{hRc}_{0}$. More precisely, $\mathcal{F}_{\left.F_{i}\right|_{L}}$ has no hRc.

Proof: Suppose, by contradiction, that there is a hRc $\mathcal{A}$ of $\mathcal{F}_{\left.F_{i}\right|_{L}}$. We will adapt the argument of Theorem 4(i) of [10] to construct a sequence $p_{n} \rightarrow \infty$ in $L$ such that $F_{i}\left(p_{n}\right) \rightarrow c \in \mathbb{R}$ but $\left\|D\left(\left.F_{i}\right|_{L}\right)\left(p_{n}\right)\right\| \rightarrow 0$, when $n \rightarrow \infty$. This contradiction with PS condition of $\left.F_{i}\right|_{L}$ will prove the proposition.

Let $L_{p}$ and $L_{q}$ the leaves of $\mathcal{F}_{\left.F_{i}\right|_{L}}$ containing the non compact edges of $\mathcal{A}$ and let $c \in \mathbb{R}$ such that $F_{i}\left(L_{p}\right)=F_{i}\left(L_{q}\right)=c$. Let $\Sigma_{p}$ and $\Sigma_{q}$ be one-sided compact transversal sections of $L_{p}$ and $L_{q}$ passing through $p$ and $q$, respectively, and contained at the canonical region $\Omega_{0}$ of $\left.\mathcal{F}_{F_{i}}\right|_{L}$ between the leaves $L_{p}$ and $L_{q}$. Furthermore, we can choose $\Sigma_{p}$ and $\Sigma_{q}$ in such a way that the Poincaré map $\pi: \Sigma \backslash\{p\} \rightarrow \Sigma \backslash\{q\}$ is defined and satisfies $\lim _{x \rightarrow p} \pi(x)=q$. Taking $p_{0} \in \Sigma_{p}$ and $q_{0} \in \Sigma_{q}$ such that $\pi\left(p_{0}\right)=q_{0}$, denote by $\Omega$ the open subset of $\Omega_{0}$ determined by the segment of leaf joining $p_{0}$ and $q_{0}$, the segments of the two one-sided transversal sections $\left[p, p_{0}\right] \subset \Sigma_{p}$ and $\left[q_{0}, q\right] \subset \Sigma_{q}$, and the two leaves $L_{p}$ and $L_{q}$ (see Figure 2).

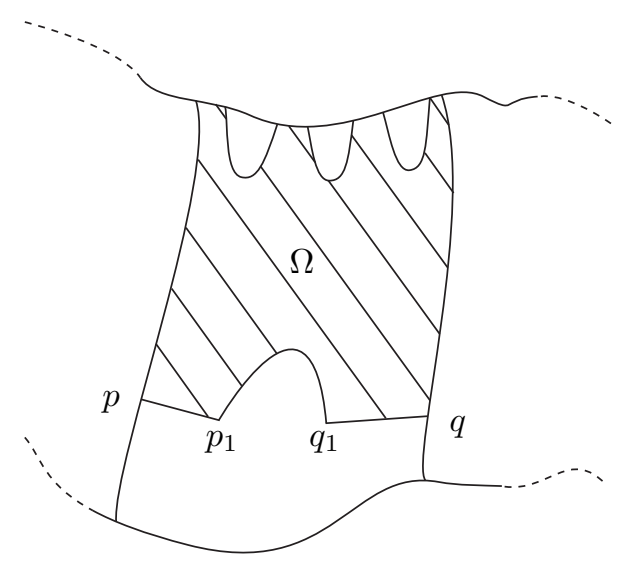

Figure 2. The subset $\Omega \subset \Omega_{0}$.

Observe that $\Omega$ is an unbounded set and that $F_{i}$ assume values different than $c$ in $\Omega$, so we can assume that $F_{i}$ takes values strictly less than $c$ 
on it. In fact, concerning the canonical region $\Omega_{0}$, the function $F_{i}$ assume the value $c$ only in the leaves $L_{p}, L_{q}$ and perhaps in other separatrices of $\Omega_{0}$.

Consider an increasing sequence $\left(\delta_{n}\right)$ in $\mathbb{R}$ such that $\delta_{n} \rightarrow \infty$ when $n \rightarrow \infty$ and the corresponding sequence of 3 -dimensional balls $B\left(\delta_{n}\right)$ centered at the origin of $\mathbb{R}^{3}$ with radius $\delta_{n}$. Note that this sequence can be chosen in a such way that for each $n$ we can choose a point $r_{n}$ lying in $\Omega \cap\left(B\left(\delta_{n}\right) \backslash B\left(\delta_{n-1}\right)\right)$ and with $F_{i}\left(r_{n}\right)$ being a strictly increasing sequence. Since the leaves containing the sequence $r_{n}$ accumulate at $L_{p}$ and $L_{q}$, we have $F_{i}\left(r_{n}\right) \rightarrow c$ when $n \rightarrow \infty$. For each point $n$, consider the solution $\phi_{t}\left(r_{n}\right)$ of the vector field $D\left(\left.F_{i}\right|_{L}\right)$ passing through $r_{n}$ in $t=0$ and defined for $t \in(-1 / 4,1 / 4)$. If such a solution is not entirely contained at $\Omega$, change $r_{n}$ by the point $\phi_{-1 / 4}\left(r_{n}\right)$ which is in $\Omega$ and by construction the solution of the vector field $D\left(\left.F_{i}\right|_{L}\right)$ passing through it belongs now to $\Omega$ for all $t \in(-1 / 4,1 / 4)$. For simplicity, let us rename all these points again by $r_{n}$.

Let $\gamma: \mathbb{R} \rightarrow \Omega$ be a $C^{1}$ parametrized curve that coincides with $\phi_{t}\left(r_{n}\right)$ around each point $r_{n}$. We can assume that $\gamma(n)=r_{n}$ for all $n \geq 0$ and that the parametrization is by arc length, i.e. $\left\|\gamma^{\prime}(t)\right\|=1$.

Applying the Mean Value Theorem to $g(t)=F_{i}(\gamma(t))$ around each $n$ in the intervals $I_{n}=(n-1 / 4, n+1 / 4)$ we may conclude that there is a $c_{n}$ such that:

$$
g(n+1 / 6)-g(n)=\frac{1}{6} g^{\prime}\left(c_{n}\right)
$$

Note that

$$
\begin{aligned}
g^{\prime}\left(c_{n}\right) & =D F_{i}\left(\gamma\left(c_{n}\right)\right)\left(\gamma^{\prime}\left(c_{n}\right)\right) \\
& =\left\langle\nabla F_{i}\left(\gamma\left(c_{n}\right)\right), \gamma^{\prime}\left(c_{n}\right)\right\rangle=\left\|\nabla F_{i}\left(\gamma\left(c_{n}\right)\right)\right\|\left\|\gamma^{\prime}\left(c_{n}\right)\right\| \cos \theta_{n},
\end{aligned}
$$

where $\theta_{n}$ is the angle between the vectors $\nabla F_{i}\left(\gamma\left(c_{n}\right)\right)$ and $\gamma^{\prime}\left(c_{n}\right)$.

Since $g(n+1 / 6)-g(n) \rightarrow 0$, we have that $g^{\prime}\left(c_{n}\right) \rightarrow 0$ when $n \rightarrow \infty$. As $\left\|\gamma^{\prime}\left(c_{n}\right)\right\|=1$, we have two possibilities: $\left\|\nabla F_{i}\left(\gamma\left(c_{n}\right)\right)\right\| \rightarrow 0$ or $\theta_{n} \rightarrow \pi / 2$. The second case means that $\nabla F_{i}\left(\gamma\left(c_{n}\right)\right)$ is becoming orthogonal to $T_{\gamma\left(c_{n}\right)} L$ (since $\gamma^{\prime}\left(c_{n}\right)$ is the solution of the orthogonal projection of $\nabla F_{i}(p)$ at $\left.T_{p} L\right)$. In any case we will have $D\left(\left.F_{i}\right|_{L}\right)\left(\gamma\left(c_{n}\right)\right) \rightarrow 0$, since this vector is the orthogonal projection of $\nabla F_{i}(p)$ at $T_{p} L$. Hence, the sequence $p_{n}=\gamma\left(c_{n}\right)$ satisfies $p_{n} \rightarrow \infty, F_{i}\left(p_{n}\right) \rightarrow c$ but $\left\|D\left(\left.F_{i}\right|_{L}\right)\left(p_{n}\right)\right\| \rightarrow 0$, as desired.

Another consequence of Proposition 4.2 and the proof of Proposition 4.4 is: if $\left.F_{i}\right|_{L}: L \rightarrow \mathbb{R}$ satisfy the PS condition, for all $L \in \mathcal{F}_{F_{j}}$, $j \neq i$, then $\mathcal{F}_{F_{i}}$ has no $\mathrm{hRc}_{2}$. 


\section{References}

[1] H. Bass, E. H. Connell, And D. Wright, The Jacobian conjecture: reduction of degree and formal expansion of the inverse, Bull. Amer. Math. Soc. (N.S.) 7(2) (1982), 287-330. DOI: 10.1090/ S0273-0979-1982-15032-7.

[2] A. BiaŁynicki-Birula and M. Rosenlicht, Injective morphisms of real algebraic varieties, Proc. Amer. Math. Soc. 13(2) (1962), 200-203. DOI: $10.2307 / 2034464$.

[3] F. Braun and J. R. Dos Santos Filho, The real Jacobian conjecture on $\mathbb{R}^{2}$ is true when one of the components has degree 3 , Discrete Contin. Dyn. Syst. 26(1) (2010), 75-87. DOI: 10.3934/ dcds.2010.26.75.

[4] M. Chamberland and G. Meisters, A mountain pass to the Jacobian conjecture, Canad. Math. Bull. 41(4) (1998), 442-451. DOI: $10.4153 /$ CMB-1998-058-4.

[5] S. Cynk AND K. RuseK, Injective endomorphisms of algebraic and analytic sets, Ann. Polon. Math. 56(1) (1991), 29-35.

[6] J. J. DuistermaAt And L. HöRmander, Fourier integral operators. II, Acta Math. 128(3-4) (1972), 183-269. DOI: 10.1007/ BF02392165.

[7] A. VAN DEN Essen, "Polynomial automorphisms and the Jacobian conjecture", Progress in Mathematics 190, Birkhäuser Verlag, Basel, 2000. DOI: 10.1007/978-3-0348-8440-2.

[8] A. Fernandes, C. Gutierrez, and R. Rabanal, Global asymptotic stability for differentiable vector fields of $\mathbb{R}^{2}, J$. Differential Equations 206(2) (2004), 470-482. DOI: 10.1016/j.jde. 2004. 04.015.

[9] C. Gutierrez, A solution to the bidimensional global asymptotic stability conjecture, Ann. Inst. H. Poincaré Anal. Non Linéaire 12(6) (1995), 627-671.

[10] C. Gutierrez, X. Jarque, J. Llibre, and M. A. Teixeira, Global injectivity of $C^{1}$ maps of the real plane, inseparable leaves and the Palais-Smale condition, Canad. Math. Bull. 50(3) (2007), 377-389. DOI: $10.4153 / \mathrm{CMB}-2007-036-0$.

[11] C. Gutierrez and C. Maquera, Foliations and polynomial diffeomorphisms of $\mathbb{R}^{3}$, Math. Z. 262(3) (2009), 613-626. DOI: 10.1007/s00209-008-0393-7.

[12] C. Gutierrez, J. Martínez-Alfaro, and J. Venato-Santos, Plane foliations with a saddle singularity, Topology Appl. 159(2) (2012), 484-491. DOI: 10.1016/j.topol.2011.09.023. 
[13] J. Hadamard, Sur les transformations ponctuelles, Bull. Soc. Math. France 34 (1906), 71-84.

[14] Z. JeloneK, Geometry of real polynomial mappings, Math. Z. 239(2) (2002), 321-333. DOI: 10.1007/s002090100298.

[15] C. Maquera and J. Venato-Santos, Foliations and global injectivity in $\mathbb{R}^{n}$, Bull. Braz. Math. Soc. (N.S.) 44(2) (2013), 273-284. DOI : $10.1007 / \mathrm{s} 00574-013-0013-y$.

[16] L. Markus and H. YamaBe, Global stability criteria for differential systems, Osaka Math. J. 12 (1960), 305-317.

[17] S. Nollet And F. Xavier, Global inversion via the Palais-Smale condition, Discrete Contin. Dyn. Syst. 8(1) (2002), 17-28.

[18] C. OleCH, On the global stability of an autonomous system on the plane, Contributions to Differential Equations 1 (1963), 389-400.

[19] C. F. B. Palmeira, Open manifolds foliated by planes, Ann. Math. (2) 107(1) (1978), 109-131.

[20] S. Pinchuk, A counterexample to the strong real Jacobian conjecture, Math. Z. 217(1) (1994), 1-4. DOI: $10.1007 /$ BF02571929.

[21] R. Plastock, Homeomorphisms between Banach spaces, Trans. Amer. Math. Soc. 200 (1974), 169-183. DOI: 10.1090/S0002-99471974-0356122-6.

[22] R. Rabanal, Center type performance of differentiable vector fields in the plane, Proc. Amer. Math. Soc. 137(2) (2009), 653-662. DOI: 10.1090/S0002-9939-08-09686-X.

[23] P. J. RABIER, On global diffeomorphisms of Euclidean space, Nonlinear Anal. 21(12) (1993), 925-947. DOI : 10.1016/0362-546X(93) 90117-B.

[24] P. J. RABIER, Ehresmann fibrations and Palais-Smale conditions for morphisms of Finsler manifolds, Ann. of Math. (2) 146(3) (1997), 647-691. DOI : $10.2307 / 2952457$.

[25] J. R. Dos SAntos FilHo, Injective mappings and solvable vector fields of Euclidean spaces, Topology Appl. 136(1-3) (2004), 261-274. DOI: 10.1016/j.topol.2003.05.001.

[26] J. R. Dos Santos Filho And J. Tavares, Injective mappings and solvable vector fields, An. Acad. Brasil. Ciênc. 82(3) (2010), 555-559. DOI: 10.1590/S0001-37652010000300002.

[27] E. A. DE B. E Silva And M. A. TeixeirA, A version of Rolle's theorem and applications, Bol. Soc. Brasil. Mat. (N.S.) 29(2) (1998), 301-327. DOI: $10.1007 / \mathrm{BF} 01237653$. 
Francisco Braun:

Departamento de Matemática

Universidade Federal de São Carlos

Rod. Washington Luis, Km 235 - C.P. 676

13565-905 São Carlos, SP

Brasil

E-mail address: franciscobraun@dm.ufscar.br

Jean Venato-Santos:

Faculdade de Matemática

Universidade Federal de Uberlândia

Av. João Naves de Ávila 2121

1F120 - CEP: 38408-100 Uberlândia

Brazil

E-mail address: jvenatos@famat.ufu.br 